\title{
COLD ATOMS AND PRECISION SENSORS IN SPACE
}

\author{
W. Ertmer ${ }^{1}$, E. Rasel ${ }^{1}$, C. Salomon ${ }^{2}$, S. Schiller ${ }^{3}$, G.M. Tino ${ }^{4}$ and L. Cacciapuoti ${ }^{5}$ \\ ${ }^{1}$ IQO Hannover, ${ }^{2}$ ENS Paris, ${ }^{3}$ Heinrich-Heine-Universität Düsseldorf, ${ }^{4}$ University of Firenze, ${ }^{5}$ European Space Agency
}

ooling atoms, which means slowing them down to very

low velocity, enables scientists to realise accurate physical measurements based on quantum transitions between atomic energy levels. A well-known spin-off is the astonishing precision of atomic clocks. Measurement accuracy improves as the interaction time between atoms and excitation field increases. Laser cooling can reduce the velocity of the atoms and increase the interaction times, but it cannot cope with Earth gravity without perturbing the measurement. In ground-based laboratories, atomic fountains help to circumvent gravity and extend the measurement duration. This naturally led scientists to envisage experimenting with ultra-cold atoms in space where long and unperturbed evolution times are possible in a freely falling laboratory. Such an environment enables one to exploit the potential of cooling atoms close to stand still permitting:

- extension of the measurement time by one to three orders of magnitude in a perturbation-free environment;

- quantum evolution unbiased by gravity;

- reduction of the kinetic energy by up to three orders of magnitude, down to the sub-pico-Kelvin regime.

\section{Atomic clocks}

A pioneering step will take place in 2013, when the Columbus module will receive the Atomic Clock Ensemble in Space (ACES) payload [1], carrying ultra-stable atomic clocks and a high-precision time transfer system. Using this accurate time reference in space, the ESA-led ACES mission will perform new tests of general relativity, search for possible minute violations of Einstein's equivalence principle, and develop several applications in Earth observation and geodesy.

Clocks are basic instruments for science, technology and industry. For several decades, the most accurate and stable clocks have been atomic clocks using the hyperfine transition of the neutral Cs atom at $9.1 \mathrm{GHz}$ as quantum reference. This transition also defines the unit of time. Every Europhysics News reader has played with a GPS receiver! In this global navigation system, each GPS satellite carries a set of precise atomic clocks. The orbiting satellites send electromagnetic timing signals to re- ceivers on Earth that can determine their position with submeter accuracy by a simple triangulation method. Compared to GPS clocks, the time scale provided by ACES in space will have a hundred-fold increase in precision. Indeed in the PHARAO clock, developed by the French space agency CNES in the frame of the ACES mission, caesium atoms are cooled to a temperature of $1 \mu \mathrm{K}$, corresponding to velocities of about $7 \mathrm{~mm} / \mathrm{s}$ (Fig 1). For free-falling atoms, these low velocities cannot be maintained on the Earth surface because of the gravity acceleration. In space, there is no such limit and atoms in free expansion can be probed for several seconds. In this way, we expect the space clock to reach a relative frequency stability and accuracy of 1 part in $10^{16}$, which corresponds to an error of less than one second over 300 million years.

While the accuracy of cold Cs atom clocks is already very high, recent advances in laser and quantum technology have opened the way to the realization of even more accurate and stable clocks. In optical clocks, the reference oscillator is a laser whose frequency is continuously controlled by comparison with the atomic transition. It is expected that clocks operating in the optical domain of the electromagnetic spectrum rather than in the microwave domain (with a $-10^{5}$ times larger frequency) will bring at least two orders of magnitude improvement, reaching the $10^{-18}$ stability and accuracy level.

Two development lines are currently investigated: optical clocks based on a single atomic ion in an electrodynamic trap and optical lattice clocks, based on ensembles of $\sim 10^{4}$ neutral atoms trapped in the periodic potential produced by an intense laser. Present accuracy of ion clocks reaches a few parts in $10^{17}$ [2], while lattice clocks have been evaluated to one part in $10^{16}$ [3]. Both laboratory clock types exhibit frequency instability significantly lower than that of Cs clocks, reaching levels as low as $1 \cdot 10^{-16}$ after few hours of averaging.

When operated in space, optical clocks can enable high-precision experiments, e.g. measurements of the gravitational redshift due to various solar-system bodies, of the Shapiro gravitational time delay, and accurate tests of the space-time independence of fundamental constants. One particularly attractive

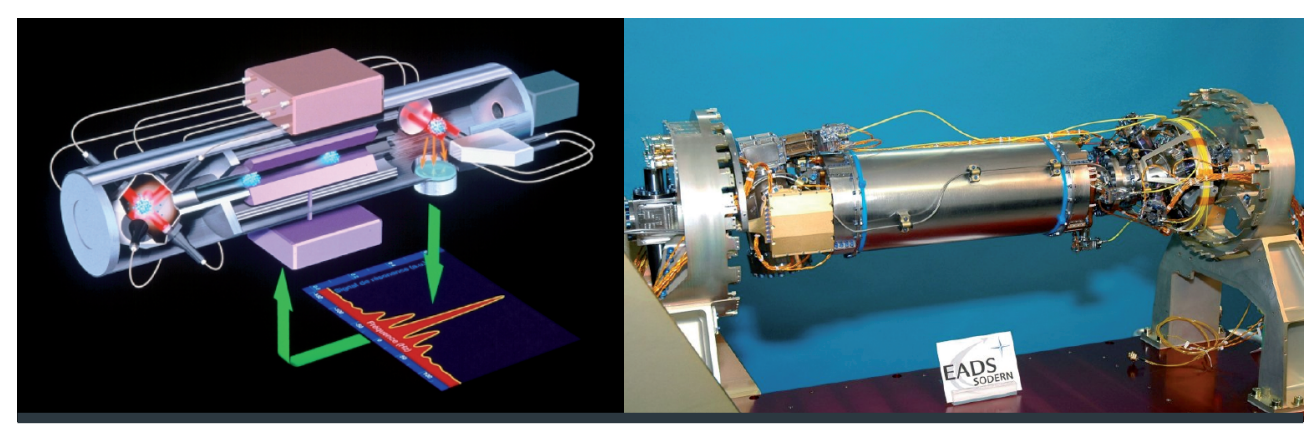

4 FIG. 1: (Left) Working principle of the PHARAO clock (Projet d'Horloge Atomique à Refroidissement d'Atomes en Orbite); Cs atoms are launched in free flight along the ultra-high vacuum tube where they are probed on the clock transition by the resonant field in a microwave cavity. (Right) Engineering model of the clock interrogation tube; The clock is presently under test at CNES premises in Toulouse. 
- application of clocks in space is a high-precision mapping of the gravitational potential at the Earth's surface. This technique, which will be first demonstrated by the ACES mission, is based on the precision measurement of the gravitational red-shift between clocks on the ground, continuously compared via a master clock in space. Implementing optical clocks in space requires a concerted development effort of research groups, industry, and space agencies. Size, mass, power consumption, reliability, and performance of the clock shall reach a level compatible with the individual applications. The Space Optical Clock (SOC) project, supported by ESA and national space agencies, is developing this technology, currently focusing research efforts towards a transportable prototype of an optical clock based on $\mathrm{Sr}$ atoms.

\section{Atom interferometry sensors}

Atomic clocks are not the only instruments which benefit from weightlessness! Clocks belong to a larger class of inertial sensors based on ultra-cold atoms and on the interference of atomic matter-waves. Inertial sensors using atom interferometry provide a new tool for the precise measurement of acceleration, rotations, and faint forces [3]. According to the principle of these sensors, the measured physical quantity is converted into a frequency, which can be measured with the highest accuracy. Atomic gravimeters, gyroscopes, and gravity gradiometers have now reached on the ground a level of performance competitive with classical instruments and surpassing them in some cases. The most important features of these instruments are represented by the precisely known calibration factor and the good long-term stability, predestining these sensors for applications in inertial references, in the definition of SI units, and for precision measurements of tiny effects on long time scales (e.g. local gravity variations...). Under microgravity conditions, atom interferometry sensors benefit from the extended measurement time even more than clocks. Indeed, acceleration sensitivity improves with the square of the measurement time and thus a ten-time longer free flight will provide a hundred times higher sensitivity.

Inertial sensors are used nowadays in many experiments probing the rotation of the Earth, its gravity and gravity gradient, and for fundamental physics tests; recent studies are also investigating applications for the astronomy of gravitational waves sources. Future experiments in these domains are challenged by the aim to measure forces in the $10^{-15}$ range of terrestrial gravity.


4 FIG. 2:

QUANTUS - a facility to produce degenerate quantum gases in the microgravity environment of the drop tower at the ZARM in Bremen. The robust and transportable setup produces Bose-Einstein condensates of ${ }^{87} \mathrm{Rb}$.
Experiments in space will exploit the full potential of these sensors. From this point of view, the Space Atom Interferometer (SAI) project coordinated by ESA intends to study applications of inertial matter-wave sensors in microgravity. SAI is pushing present performances, demonstrating this technology with a transportable sensor which will serve as a prototype for the space qualification of the final instrument. Further tests can be envisioned in drop towers or on parabolic flights, finally leading to experiments in orbit.

\section{Bose-Einstein condensation}

A leap forward in the field of atom optics has been the 1995 achievement of Bose-Einstein condensation (BEC). Cold quantum gases and, in particular, Bose-Einstein condensates are a new state of matter with very peculiar properties. BEC gives unique insights into a broad range of fundamental physics phenomena and offers prospects for novel quantum sensors [4].

The ongoing research on cooling and manipulation of atoms was and is to a great part motivated by reaching ever-new frontiers in low-temperature physics and achieving full control of these elementary quantum systems. With the current technology, atoms can be cooled to such low temperatures that Earth gravity represents a major perturbation. The present low temperature record is just below $500 \mathrm{pK}$, equivalent to the potential energy of a single $\mathrm{Rb}$ atom at a height of $5 \mathrm{~nm}$, much smaller than the typical extension of its wavefunction.

Microgravity will set the stage for innovative studies. These include thermodynamics of systems at ultra-low temperatures (pK to $\mathrm{fK}$ regime); new phase transitions such as magnetism in the quantum domain where weak forces (long-range) govern the kinetics of the ultra-cold gas; physics of dilute gases and giant matter-waves; quantum gas mixtures unbiased by gravity; highbrilliance sources of ultra-cold atoms for atom interferometry.

A facility for studying BEC properties in microgravity has been developed with DLR support for the Bremen drop-tower experiment (Fig. 2). This apparatus routinely produces BEC during free-fall from a height of about $110 \mathrm{~m}$, demonstrating an ability to well withstand decelerations that at the end of the drop can be as high as $40 \mathrm{~g}$. The next frontier is now represented by space.

In conclusion, we have briefly described the fascinating and interdisciplinary applications of atomic quantum sensors in space. Atom optics is providing us with high-precision instruments to investigate the laws of physics and improve the perception of space-time surrounding us.

\section{References}

[1] C. Salomon, L. Cacciapuoti, and N. Dimarcq, Int. J. Mod. Phys. D 16, 2511 (2007).

[2] T. Rosenband et al., Science 319, 1808 (2008).

[3] A.D. Ludlow et al., Science 319, 1805 (2008).

[4] P. Berman, Ed., Atom Interferometry (Academic Press, New York, 1997); A.D. Cronin, J. Schmiedmayer, and D.E. Pritchard, arXiv:0712.3703v1 [quant-ph] (2007).

[5] J. R. Anglin and W. Ketterle, Nature 416, 211 (2002). 